\title{
Optimal Power Allocation for Multiple ACCESS CHANNEL
}

\author{
Zouhair Al-qudah \\ Communication Engineering Department, AL-Hussein bin Talal University, \\ Ma'an, Jordan
}

\begin{abstract}
This paper considers the problem of power allocation between the two senders in the multiple access channel. Two power allocation criteria are developed. In particular, in the first criterion, the total available power is allocated between the two users such that the two users have the same achievable rate. In addition, the second criterion allocates the total available power such that the sum rate is maximized. In addition, many numerical examples are shown to show the value of power allocation and also to compare between the proposed criteria.
\end{abstract}

\section{KEYWORDS}

Multiple Access Channel, Channel Capacity, Optimal Power Allocation.

\section{INTRODUCTION}

Multiple access channel (MAC) models a communication scenario in which many users want to communication with a common receiver. For instance, consider many devices want to communicate with a base station. This communication model were investigated in many different scenarios. For example, the authors in $[1,2]$ studied the case in which the two senders transmit independent message signals to the destination. In another scenario, the case in which the two senders cooperate to transmit a common message was studied in [3,4]. Furthermore, the authors in $[6,7]$ considered the case in which the destination is affected by a third source, the interferer.

The power allocation problem is an important issue to allocate the total available power among the users based on the target criterion. For example, some authors like [10]considered the problem of power allocation problem such that all users have the same data rate. In another scenario, the authors in $[8,9]$ considered the problem of maximizing the sum rate capacity.

In this paper, the problem of allocating the total available power to the two senders in the basic Gaussian MAC channel is considered. In particular, two optimization criteria are developed. In the first optimization problem, the total available power is allocated such that the two senders can transmit to their destination using the same data rate. In addition, in the second optimization problem, the total available power is allocated such that the sum rate is maximized. Further, many numerical examples are shown to support our theoretical results.

The rest of this paper is organized as follows. The system model is introduced in Section 2. Then, two optimization problems are investigated in Section 3. Finally, the paper is concluded in Section 3.

DOI: 10.5121/ijwmn.2016.8603 


\section{Gaussian Multiple Channel Model and Capacity Region}

The MAC channel models a communication scenario in the case that at least two senders transmit to a destination, as depicted in Figure 1. Specifically, the first sender wants to transmit a signal $X_{1}$. Simultaneously, the second sender wants to transmit a signal $X_{2}$. These senders transmit their signal with power constraints $P_{1}$ and $P_{2}$, respectively. In addition, The received signal, $Y$, at the destination is given by [1]

$$
Y=a X_{1}+b X_{2}+Z
$$

where $\mathbf{a}$ and $\mathbf{b}$ are the channel gains from the first sender and the second sender to the destination, respectively. In addition, $Z$ is the additive white Gaussian noise (AWGN) signal with zero mean and variance normalized to 1 . Further, the capacity region for this channel model is given as[1]

$$
\begin{aligned}
& R_{1} \leq I\left(X_{1} ; Y \mid X_{2}\right)=C\left(a^{2} P_{1}\right) \\
& R_{2} \leq I\left(X_{2} ; Y \mid X_{1}\right)=C\left(b^{2} P_{2}\right) \\
& R_{1}+R_{2} \leq I\left(X_{1}, X_{2} ; Y\right)=C\left(a^{2} P+b^{2} P_{2}\right)
\end{aligned}
$$

where $C(x)=0.5 \log _{2}(1+x)$ bit per second (bps). Moreover, $R_{1}$ is the achievable capacity of the first sender, $R_{2}$ is the achievable capacity of the second user. In this capacity region, for example, the rate $I\left(X_{1} ; Y \mid X_{2}\right)=C\left(a^{2} P_{1}\right)$ can be achieved by decoding $X_{2}$ at first with probability goes to zero. Then, the receiver can decode the signal $X_{1}$. In addition, $I\left(X_{1}, X_{2} ; Y\right)=C\left(a^{2} P+b^{2} P_{2}\right)$ represents the sum rate of decoding both $X_{1}$ and $X_{2}$ at the destination.

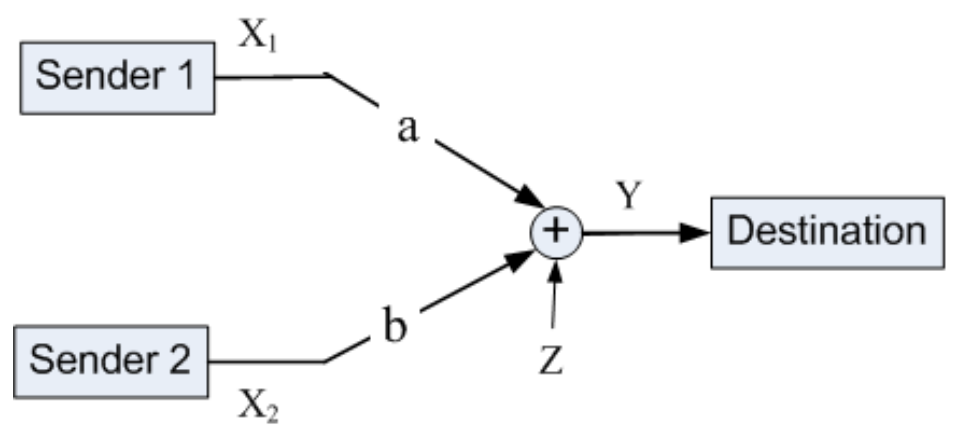

Figure 1. Gaussian Multiple Access Channel. 


\section{OPTIMAL TRANSMIT POWER ALLOCATION}

In this section, two optimal allocation criteria are investigated. In the first criterion, the total available power is allocated such that the two users have the same rate. In addition, in the second criterion, the total available power is allocated such that the sum rate is maximized.

\subsection{General Optimal Power Allocation with Data Rate Fairness}

In this subsection, the available total power is allocated to the two users such that the two users have the same data rate.

$$
\begin{array}{ll}
\max & R_{1} \\
\text { Subject to } & R_{1}=R_{2} \\
& P_{1}+P_{2}=P_{T}
\end{array}
$$

where $P_{T}$ represents the total available power. Mathematically, we need to maintain $C\left(a^{2} P_{1}\right)=C\left(b^{2} P_{2}\right)$. This condition yields that

$$
P_{1}=\left(\frac{b^{2}}{a^{2}}\right) P_{2}
$$

Further, by substituting the value of $P_{1}$ from (4) in $P_{1}+P_{2}=P_{T}$, we may get

$$
P_{2}=\left(\frac{a^{2}}{a^{2}+b^{2}}\right) P_{T}
$$

and

$$
P_{1}=\left(\frac{b^{2}}{a^{2}+b^{2}}\right) P_{T}
$$

Numerically, as shown in Figure 2, more power is allocated to the user which has the weaker channel gain with the destination. For instance, at given point like $P_{T}=15$, more power is allocated to the second transmitter as the channel gain $\mathrm{b}$ decreases. In addition, we note that while we keep $R_{1}=R_{2}$, the sum rate $R_{1}+R_{2} \leq I\left(X_{1}, X_{2} ; Y\right)=C\left(a^{2} P+b^{2} P\right)$ should be kept. 


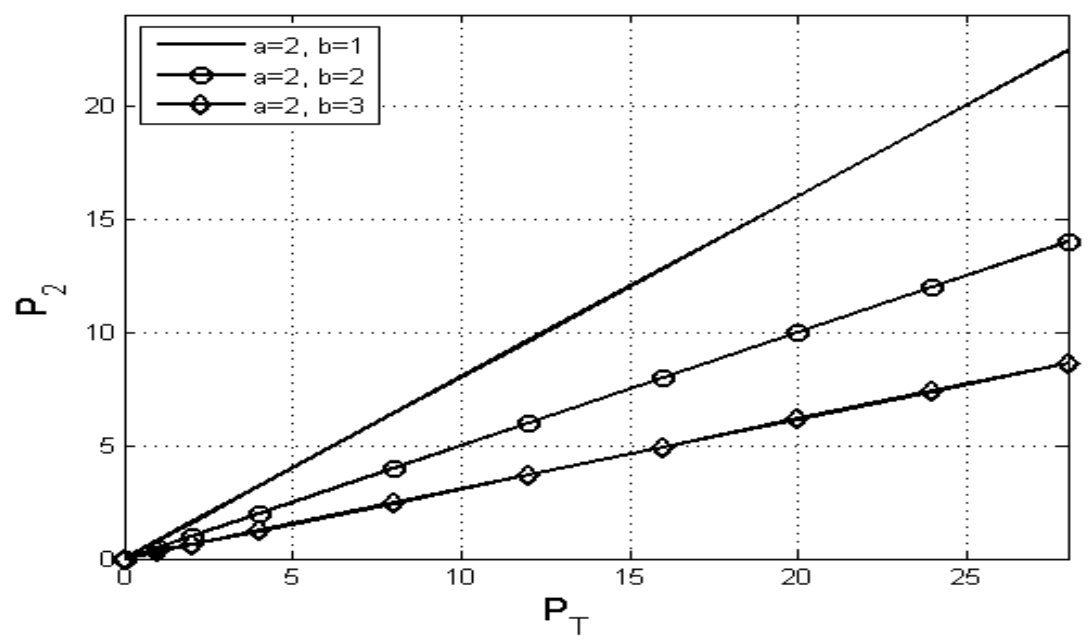

Figure 2. Power Allocated to the second user Vs. Total available power for different values of the channel gain, b.

In addition, Figure 3 shows the capacity of the second user as a function of the total available power for different values of the channel gain, $\mathbf{b}$. We remind that, in this case, both users have the same capacity. This figure shows that as the total available power increases so does the capacity of each user. In addition, as the channel gain $\mathbf{b}$ increases so does the capacity of the second user. Remember that as the channel gain $\mathbf{b}$ increases, more power will allocated to the other sender, the first sender.

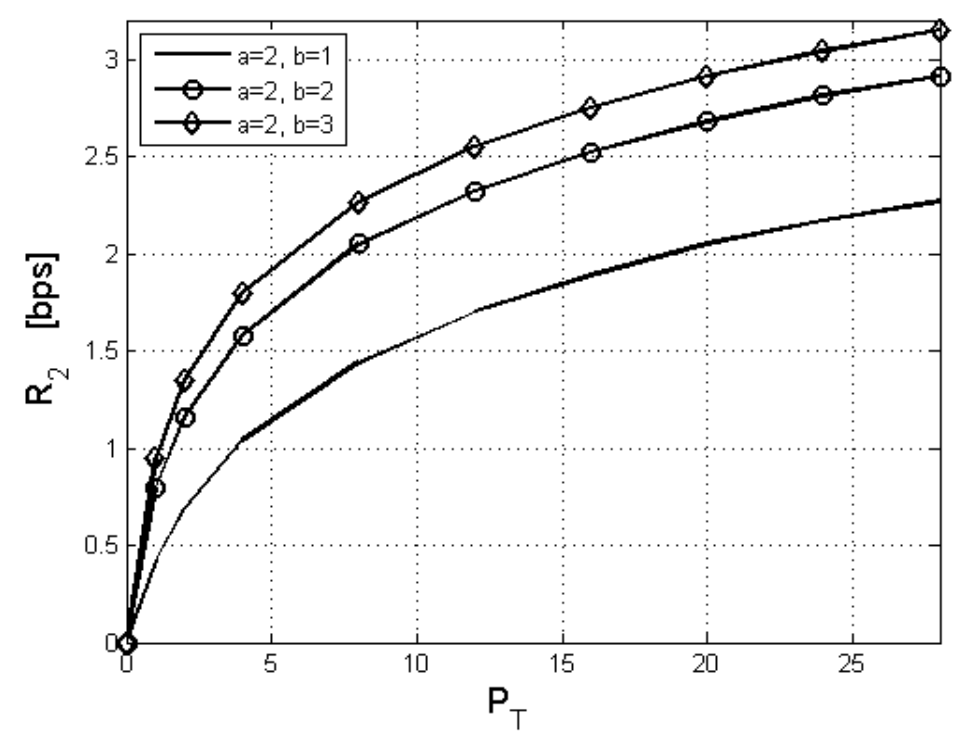

Figure 3. Capacity of the second user as a function of the total available power for different values of the channel gain, $b$. 
International Journal of Wireless \& Mobile Networks (IJWMN) Vol. 8, No. 6, December 2016

\subsection{Optimal Power Allocation with Sum Rate Maximization.}

In this subsection, the total available power is divided between the two senders such that the sum rate $\left(R_{1}+R_{2}\right)$ is maximized. This optimization problem can be modelled as

$$
\begin{array}{ll}
\max & R_{1}+R_{2} \\
\text { Subject to } & P_{1}+P_{2}=P_{T}
\end{array}
$$

In maximizing $R_{1}+R_{2} \leq C\left(a^{2} P+b^{2} P_{2}\right)$, it is enough to maximize $J=a^{2} P_{1}+b^{2} P_{2}$. By substituting the value of $P_{2}$ from $P_{1}+P_{2}=P_{T}$. Thus, we may get $J=\left(a^{2}-b^{2}\right) P_{1}+b^{2} P_{T}$. Further, we derive $J$ with respect to $P_{1}$. Therefore, $J$ gets its maximum for values like $a=b$. In this case, we may have $J=\max \left\{a^{2}, b^{2}\right\} P_{T}$. This means that the total power is allocated to the user which has the stronger channel gain with the destination.

Figure 4. shows the achievable capacity of the second user as a function of $P_{T}$ for different values of the channel gains $\mathbf{a}$ and $\mathbf{b}$. It clearly shows that the total available power is allocated to the second sender once the channel gain from the second sender to the destination is greater than the channel gain between the first user and the destination, and vice-versa.

Figure 5. compares the achievable capacity when different power allocation criteria are used. This figure clearly shows larger achievable capacity can be obtained in the case that the criterion is to maximize the sum rate. However, this option is not optimal since only one user can use the total available power.

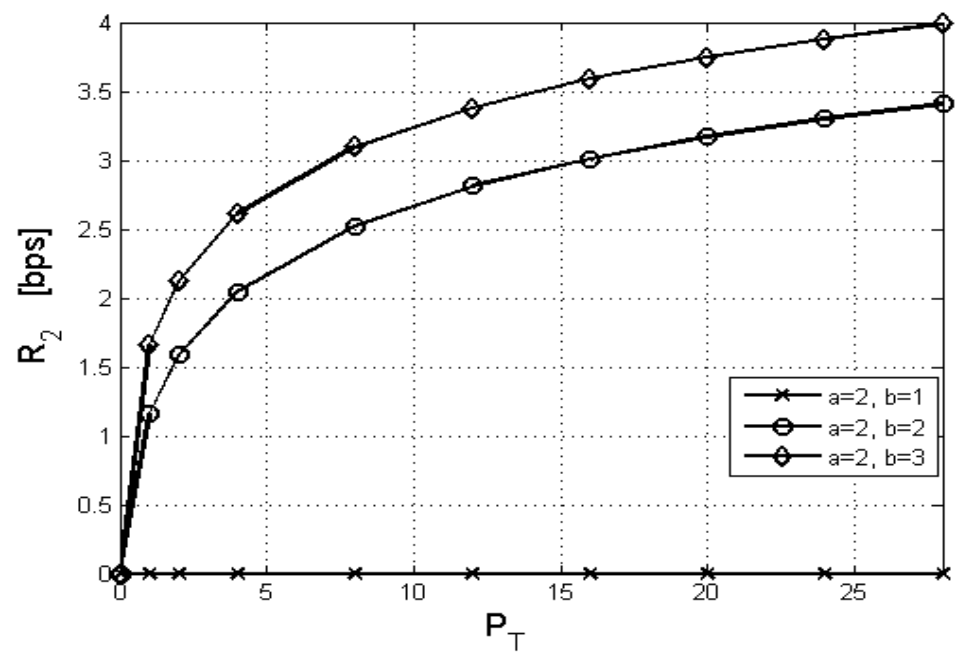

Figure 4. Capacity of the second user as a function of the total available power for different values of the channel gain, b. 


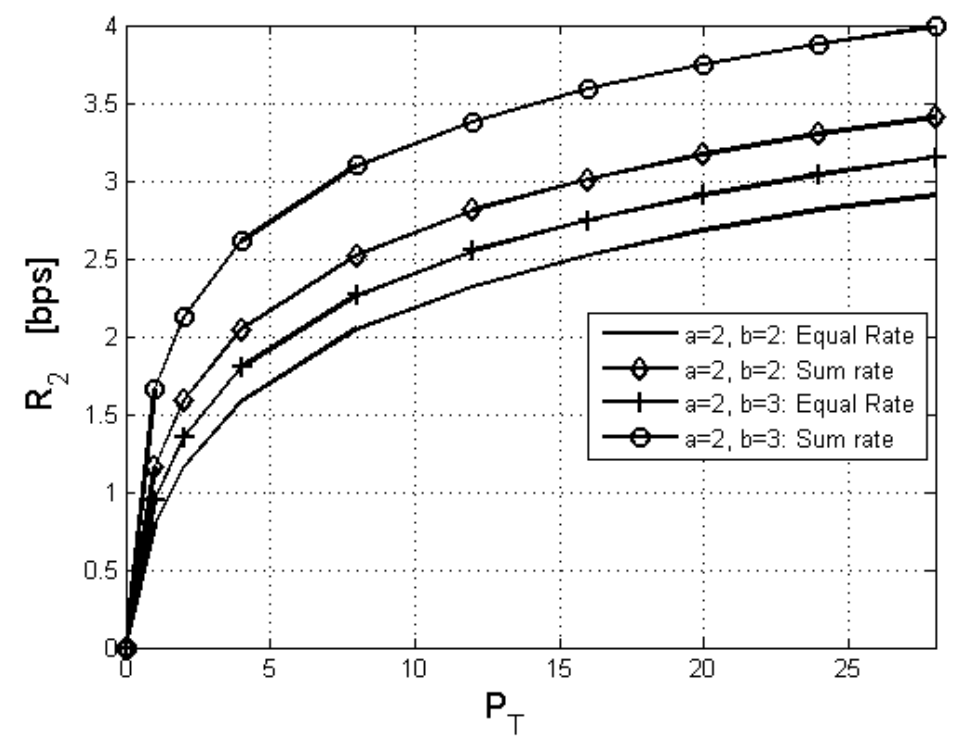

Figure 5. Comparison between the achievable capacity of the second user when different power allocation criteria are used.

\section{CONCLUSIONS}

In this paper, the problem of allocating the total available power between the two senders forming the MAC is investigated. In particular, two criteria are investigated. In the first criterion, the total power is allocated such that the two users have the same data rate. In the second criterion, the available power is allocated to maximize the sum rate. In this case, the total power has been proved to be allocated to the user with stronger channel gain with the destination. Finally, many numerical examples have been the illustrate the importance of power allocation.

\section{REFERENCES}

[1] T. Cover \& J. Thomas, (2006), Elements of Information Theory, 2nd Edition, Wiley.

[2] S. Shamai \& A. Wyner, (1997), "Information-theoretic considerations for symmetric, cellular, multiple-access fading channels. I, " IEEE Trans. Inf. Theory, vol. 43, no. 6, pp. 1877-1894.

[3] H. Asnani, \& H. Permuter,(2013) "Multiple-Access Channel With Partial and Controlled Cribbing Encoders", IEEE Trans. Inf. Theory, Vol. 59, No. 4.

[4] D. Chatterjee \& T. Wong, “Active user Cooperation in Fading Multiple-Access Channels”, IEEEMILCOM, 2008.

[5] O. Kaya \& S. Ulukus (2007), "Power Control for Fading Multiple Access Channels with User Cooperation", IEEE Trans. Wireless Communications, Vol 6, No 8.

[6] O. Kaya \& M. İşleyen, (2012), "Power control in the cognitive cooperative multiple access channel, " 46th Annual Conference on Information Sciences and Systems (CISS), Princeton, NJ. 
International Journal of Wireless \& Mobile Networks (IJWMN) Vol. 8, No. 6, December 2016

[7] N. Devroye, P. Mitran \& V. Tarokh,(2005), "Cognitive multiple access networks, " Proceedings of International Symposium on Information Theory (ISIT). Adelaide, SA.

[8] T. Park, J. Jang, O. Shin \& K. Lee,(2005), "Transmit power allocation for a downlink two-user interference channel," IEEE Communications Lett., vol. 9, no. 1. pp 13-16.

[9] H. Joudeh \& B. Clerckx,(2016), "Sum-Rate Maximization for Linearly Precoded Downlink Multiuser MISO Systems With Partial CSIT: A Rate-Splitting Approach, " IEEE Trans. Communications, Vol. 64, No. 11, pp. 4847-4861.

[10] M. Pischella \& D. Le Ruyet,(2011), “Optimal Power Allocation for the Two-Way Relay Channel with Data Rate Fairness," in IEEE Communications Lett., vol. 15, no. 9, pp. 959-961. 